\title{
Tau intricacies in disease
}

Pathological aggregation of the microtubule-associated protein tau is the underlying cause of several neurodegenerative diseases. These 'tauopathies' have very diverse neuronal phenotypes and clinical manifestations. How tau aggregation gives rise to such varied phenotypes is unclear, but it has been recently hypothesized that differently folded forms of abnormal tau might be associated with different tauopathies. Reporting in Cell, Arakhamia et al. now find that tau post-translational modifications (PTMs) and their crosstalk contribute to the formation of disease-specific intracellular tau aggregates.

Previous studies have reported several tau PTMs — which might enable tau to acquire highly ordered $\beta$-sheet structures that facilitate tau aggregation and the formation of pathological filamentous inclusions - but these modifications had not been identified in structural studies, because the treatment of tissue-derived tau removes the PTMs. Here, the authors were able to solve the structures of post-translationally modified tau filaments isolated from the brains of individuals with corticobasal degeneration (CBD) or Alzheimer disease (AD) by using a combination of cryoelectron microscopy (EM) and mass spectrometry (MS)-based proteomics.

The authors analysed denselypacked, insoluble tau inclusions from patients with $\mathrm{CDB}$ and $\mathrm{AD}$, which they found to form both straight and twisted filaments, and mapped the positions of four types of PTM acetylation, ubiquitylation, methylation and phosphorylation. Whereas some were common to CBD and $\mathrm{AD}$, the majority were distinct. Interestingly, when comparing the $\mathrm{CBD}$ and $\mathrm{AD}$ filaments, they found that although the $\beta$-strand-forming motifs in the two diseases are highly similar, the misfolding of individual tau molecules and their self-assembly into fibrils is different.

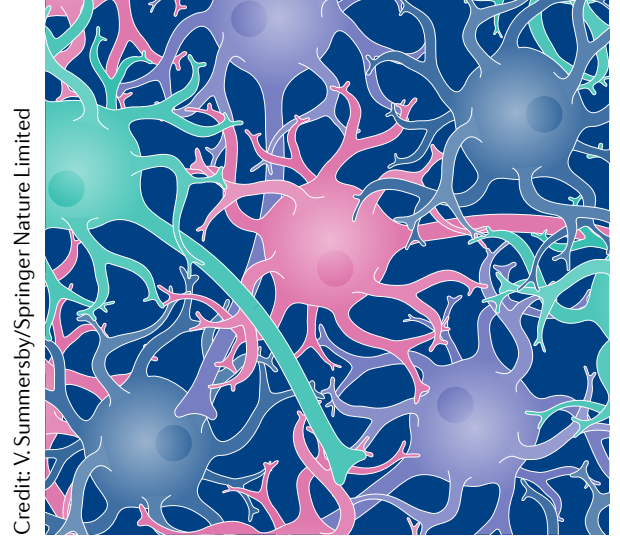

On the basis of the analyses of ubiquitylation sites, localized in the atomic models, the authors propose that ubiquitin incorporated into tau filaments in $\mathrm{CBD}$ and $\mathrm{AD}$ mediates specific inter-protofilament packing. Ubiquitin chains may provide additional contacts between tau molecules, favouring the formation and stabilization of fibril subtypes that are specific to the disease. Thus, the approach of combining cryo-EM with MS-based proteomics has the potential to reveal the role of PTMs in the onset and development of neurodegenerative diseases.

Kim Baumann

protofilament

packing

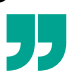

\section{Stress fibres fuel glycolysis}

Cells can sense the stiffness of the extracellular matrix (ECM) and use this information to regulate the cytoskeleton and modulate signalling. In a study published in Nature, Park et al. find a direct link between mechanical sensing, the cytoskeleton and metabolic regulation through the activity of the ubiquitin ligase TRIM21.

The authors found that human bronchial epithelial cells (HBECs) spread out less on soft ECM than on stiff ECM and had a lower level of glycolytic activity owing to a reduction in the level of isoforms of the glycolytic enzyme phosphofructokinase (PFK). However, non-small-cell lung carcinoma (NSCLC) cells, as well as HBECs transformed with oncogenes, were able to maintain a high glycolytic rate on soft ECM.

Overexpression of PFK only partially rescued the downregulation of glycolysis on soft ECM, suggesting post-translational regulation of PFK in this pathway. Proteasome inhibition or disruption of PFK ubiquitination increased PFK levels

TRIM21 is sequestered by stress fibres, which ... upregulates glycolysis in response to stiff ECM

5
and rescued glycolytic activity in cells on soft substrate. Knockdown TRIM21 increased PFK levels, and ECM and in cancer cells.

The reduced rates of glycolysis in to actin cytoskeleton architecture and specifically to reduced actin of the PFK-targeting ubiquitin ligase overexpression was sufficient to reduce PFK levels in HBEC s cultured on stiff HBECs grown on soft ECM were linked bundling. Indeed, HBECs expressing a mutant $\alpha$-actinin 1 to induce actin bundling maintained PFK expression and glycolysis even on soft substrates. Moreover, interference with myosin or actin polymerization was sufficient to inhibit glycolysis and reduce PFK expression in HBECs, but not cancer cells, on stiff ECM.

The authors further showed that TRIM21 co-localized with stress fibres — contractile actin bundles associated

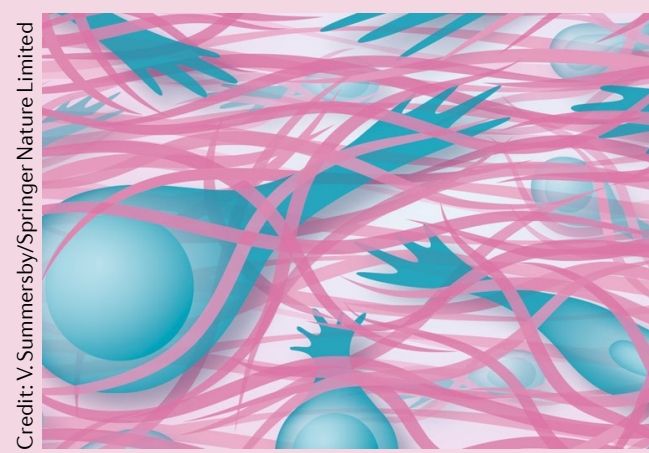

with cell spreading. Induction of stress fibres reduced cytosolic TRIM21 levels, suggesting that stress fibres sequester TRIM21.

Collectively, these results indicate that TRIM21 is sequestered by stress fibres, which prevents PFK ubiquitination and upregulates glycolysis in response to stiff ECM. These data also suggest that cancer cells can sustain high glycolytic rates through maintaining stress fibres that are insensitive to substrate stiffness.

Joseph Willson

ORIGINAL ARTICLE Park, J. S. et al. Mechanical regulation of glycolysis via cytoskeleton architecture. Nature https://doi.org/10.1038/ s41586-020-1998-1 (2020) 